\title{
ac transport through a resonant level between ferromagnetic electrodes
}

\author{
Chi-keung Lui, ${ }^{1}$ Baigeng Wang, ${ }^{2}$ and Jian Wang ${ }^{1,3, *}$ \\ ${ }^{1}$ Department of Physics, The University of Hong Kong, Pokfulam Road, Hong Kong, People's Republic of China \\ ${ }^{2}$ Department of Physics, Nanjing University, Nanjing, People's Republic of China \\ ${ }^{3}$ Key Laboratory of Materials Physics, Institute of Solid State Physics, Chinese Academy of Sciences, Hefei, Anhui, \\ People's Republic of China
}

(Received 12 February 2004; revised manuscript received 17 June 2004; published 11 November 2004)

\begin{abstract}
We report the investigation of the spin-valve effect through a resonant level between a ferromagnetic electrode in the presence of an external ac bias. We use the current conserving and gauge invariant theory developed by Büttiker to calculate the dynamic conductance. Specifically, we have calculated the tunneling magnetoresistance (TMR) ratio as a function of various system parameters such as the angle between magnetization of the left and right leads, ac frequency, and the Fermi energy. We found that the TMR ratio can be modulated by ac frequency. At large frequency, the TMR ratio can be negative.
\end{abstract}

DOI: 10.1103/PhysRevB.70.205316

PACS number(s): 73.23.Ad, 73.40.Gk, 72.10.Bg

\section{INTRODUCTION}

Quantum transport in tunnel magnetoresistance (TMR) systems provides an exciting arena for various new spinrelated phenomena such as spin-valve effect..$^{1,2}$ Due to the large TMR ratio produced at room temperature ${ }^{3}$ this research field has attracted much attention in recent years. Basically TMR (Ref. 2) arises as a result of the mismatch of the energy levels in both ferromagnetic electrodes due to the change of the relative orientation of the magnetic moments. The region between the electrodes is nonmagnetic such as an insulating layer or a double barrier structure. While the dc transport properties of ferromagnetic-nonmagnetic-ferromagnetic ( $F$ $-N-F$ ) systems have been investigated extensively, ${ }^{4-16}$ less attention has been paid to the corresponding ac case. ${ }^{17}$ As pointed out by Büttiker et al. ${ }^{18}$ the transport property a timedependent system is more complicated to study because one has to include the effect of the charge build up due to the internal Coulomb interaction inside the scattering region. This charge build up gives rise the displacement current which makes the total current conserve in the ac case. Given the fact that most of the spintronic devices operate under ac signals, there is a need to examine the transport behavior of a $F-N-F$ tunnel junction under the influence of an external ac voltage. In this work, we consider the dynamic spin valve effect through a resonant between two ferromagnetic electrodes. In particular, with the help of nonequilibrium Green functions method, we calculate the dynamic conductance as a function of the angle between magnetization of two electrods at finite frequency using a phenomenological theory first developed by Büttiker et al.. ${ }^{18}$ We find that the TMR ratio can be modulated by ac frequency. At large frequency, the TMR ratio can be negative.

The rest of this paper is organized as follows. In Sec. II, we present the general theory by deriving an expression of dynamic conductance for TMR junctions. In Sec. III, we apply our theory for various situations and a summary is given at the end.

\section{THEORY}

The TMR device that we consider consists of a scattering region with a resonant level connected by two ferromagnetic electrodes. The magnetic moment $M$ of the left electrode lies in the $z$ direction while the moment of the right electrode points at an angle $\theta$ with respect to the $z$ axis in the $x-z$ plane. Due to the spin-flip mechanism the system is not spin conserving. Under an external ac voltage $v_{\alpha} \cos \omega t$ that is applied at lead $\alpha$, the Fermi energy $\epsilon_{k \alpha}$ of lead $\alpha$ varies with time and is written as $\epsilon_{k L}(t)=\epsilon_{k L}+q v_{\alpha} \cos \omega t$. We want to calculate the dynamical response of the system to the external ac bias. Thus we use the following Hamiltonian to model our system:

$$
H=H_{L}+H_{R}+H_{0}+H_{T}
$$

where $H_{L}$ and $H_{R}$ describe the left and right electrodes where an ac bias is applied,

$$
H_{L}=\sum_{k} \psi_{k L}^{\dagger}\left[\epsilon_{k L}(t)+\sigma \cdot \mathbf{M}_{L}\right] \psi_{k L}=\sum_{k \sigma}\left[\epsilon_{k L}(t)+\sigma M\right] c_{k L \sigma}^{\dagger} c_{k L \sigma}
$$

and

$$
\begin{aligned}
H_{R} & =\sum_{k} \psi_{k R}^{\dagger}\left[\epsilon_{k R}(t)+\sigma \cdot \mathbf{M}_{R}\right] \psi_{k R} \\
& =\sum_{k \sigma}\left[\epsilon_{k R}(t)+\sigma M \cos \theta\right] c_{k R \sigma}^{\dagger} c_{k R \sigma}+\sum_{k \sigma} M \sin \theta c_{k R \sigma}^{\dagger} c_{k R \bar{\sigma}},
\end{aligned}
$$

where $\psi_{k \alpha}=\left(\begin{array}{l}c_{k \alpha \uparrow} \\ c_{k \alpha \downarrow}\end{array}\right)$. In Eq. (1), $H_{0}$ describes the scattering region with resonant levels at $\epsilon_{n}$,

$$
H_{0}=\sum_{n \sigma} \epsilon_{n} d_{n \sigma}^{\dagger} d_{n \sigma} .
$$

$H_{T}$ models the coupling between electrodes and the scattering region with hopping matrix $T_{k a n}$. To simplify the analysis, we assume the hopping matrix to be independent of spin index, hence 


$$
H_{T}=\sum_{k \alpha n \sigma}\left[T_{k \alpha n} c_{k \alpha \sigma}^{\dagger} d_{n \sigma}+\text { c.c. }\right]
$$

In these expressions $c_{k \alpha \sigma}^{\dagger}$ (with $\sigma=\uparrow, \downarrow$ or \pm 1 and $\bar{\sigma}=-\sigma$ ) is the creation operator of electrons with spin index $\sigma$ inside the $\alpha$ lead. Similarly $d_{n \sigma}^{\dagger}$ is the creation operator of electrons with spin $\sigma$ at energy level $n$ for the scattering region. In writing down Eqs. (2) and (3), we have made a simplification that the value of molecular field $M$ is the same for the two leads, thus the spin-valve effect is obtained ${ }^{2}$ by varying the angle $\theta$. Essentially, $M$ mimics the difference of density of states (DOS) between spin up and down electrons ${ }^{2}$ in the electrodes. Using the Bogoliubov transformation ${ }^{14}$

$$
\begin{gathered}
c_{k R \sigma}=\cos (\theta / 2) C_{k R \sigma}-\sigma \sin (\theta / 2) C_{k R \bar{\sigma}}, \\
c_{k R \sigma}^{\dagger}=\cos (\theta / 2) C_{k R \sigma}^{\dagger}-\sigma \sin (\theta / 2) C_{k R \bar{\sigma}}^{\dagger},
\end{gathered}
$$

$H_{R}$ can be diagonalized. We have

$$
H_{\alpha}=\sum_{k \sigma}\left[\epsilon_{k \alpha}(t)+\sigma M\right] C_{k \alpha \sigma}^{\dagger} C_{k \alpha \sigma}
$$

with $\alpha=L, R$. The spin-flip term in $H_{R}$ has been transformed into the the coupling Hamiltonian between the scattering region and the leads

$$
\begin{aligned}
H_{T}= & \sum_{k n \sigma}\left[T_{k L n} C_{k L \sigma}^{\dagger} d_{n \sigma}+T_{k R n}\left(\cos \frac{\theta}{2} C_{k R \sigma}^{\dagger}-\sigma \sin \frac{\theta}{2} C_{k R \bar{\sigma}}^{\dagger}\right) d_{n \sigma}\right. \\
& + \text { c.c. }] .
\end{aligned}
$$

With this new Hamiltonian, we are able to calculate the ac current and hence the dynamic conductance using the standard nonequilibrium Green's function (NEGF) method. ${ }^{19}$ Briefly, we start from the equation of motion method

$$
I_{\alpha}=d N_{\alpha} / d t=-i\left\langle\left[N_{\alpha}, H\right]\right\rangle
$$

and then define the lesser and greater NEGF $G^{<}$and $G^{>}$with properly contour ordered operators, and apply the theorem of analytic continuation ${ }^{20}$ so that the contour Green's functions are extended to the real time axis. This standard and widely used NEGF technique allows us to obtain the exact expression for the dynamic conductance $G_{\alpha \beta}^{c}$ (its expression will be given below). ${ }^{21}$ However, the dynamic conductance obtained this way will not satisfy the current conservation condition. This is because in Eq. (6) only the conduction current is considered and the displacement current has been left out. The physical origin of the displacement current is due to the charge accumulation in the central scattering region which has to be solved through Poisson equation. To avoid solving Poisson equation we consider the Coulomb interaction implicitly by using the theory developed by Büttiker et al. ${ }^{18}$ which conserves the current by partitioning the total displacement current into each lead. In the language of the NEGF, the dynamic conductance is ${ }^{23}$

$$
G_{\alpha \beta}=G_{\alpha \beta}^{c}-G_{\beta}^{d} \frac{\sum_{\gamma} G_{\alpha \gamma}^{c}}{\sum_{\gamma} G_{\gamma}^{d}}
$$

where

$$
\begin{aligned}
G_{\alpha \beta}^{c}(w)= & -q \int \frac{d E}{2 \pi} \operatorname{Tr}\left[\bar{g}_{\beta}^{<}\left(\Sigma_{0 \alpha}^{a}-\bar{\Sigma}_{0 \alpha}^{r}\right)+\bar{g}_{\beta}^{r} \Sigma_{0 \alpha}^{<}-\bar{\Sigma}_{0 \alpha}^{<} \bar{g}_{\beta}^{a}\right. \\
& \left.+\left(\bar{G}_{0}^{r} \bar{\sigma}_{\alpha}^{<}-\bar{\sigma}_{\alpha}^{<} G_{0}^{a}+\bar{G}_{0}^{<} \bar{\sigma}_{\alpha}^{a}-\bar{\sigma}_{\alpha}^{r} G_{0}^{<}\right) \delta_{\alpha \beta}\right]
\end{aligned}
$$

is the dynamic conductance due to the contribution from the conduction current and

$$
G_{\alpha}^{d}(\omega)=-q \omega \int \frac{d E}{2 \pi} \operatorname{Tr}\left[g_{\alpha}^{<}(E+\omega, E)\right]
$$

is the contribution from the displacement current. Here we have used the abbreviation $g$ as $g(E)$ and $\bar{g}$ as $g(E+\omega)$ and similarly for other quantities. ${ }^{23}$ In the above equation, $G^{r, a,<}$ and $\Sigma^{r, a,<}$ are the equilibrium Green's function and selfenergy, respectively. $\bar{\sigma}^{r, a},<$ are the first-order correction to the self-energy components due to the ac signal $\bar{\sigma}_{\alpha}^{<}$ $=(i q / \omega)\left[\Gamma_{\alpha} f-\bar{\Gamma}_{\alpha} \bar{f}\right]$ and $\bar{\sigma}_{\alpha}^{r, a}=(q / \omega)\left[\Sigma_{\alpha}^{r, a}-\bar{\Sigma}_{\alpha}^{r, a}\right]$, where $f(E)$ is the equilibrium Fermi distribution function. The first-order correction to the retarded, advanced and lesser Green's functions are $\bar{g}_{\alpha}^{r, a}=\bar{G}^{r, a} \bar{\sigma}_{\alpha}^{r, a} G^{r, a}$ and $\bar{g}_{\alpha}^{<}=\bar{G}^{r} \bar{\sigma}_{\alpha}^{<} G^{a}+\bar{G}^{r} \bar{\sigma}_{\alpha}^{r} G^{<}$ $+\bar{G}^{<} \bar{\sigma}_{\alpha}^{a} G^{a}$, respectively. All of these equations are expressed in terms of $2 \times 2$ matrices (in spin space) because the spin degree of freedom manifests itself in the $F-N-F$ system. To simplify the calculation we use the wideband approximation $^{19}$ so that the self-energy does not depend on the energy. As a result, $\bar{\sigma}^{r, a}$ and $\bar{g}^{r, a}$ are zero. This results in the vanishing of all retarded and advanced small-signal components (i.e., Green functions and self-energy) and so Eq. (8) simplifies as follows:

$$
G_{\alpha \beta}^{c}(w)=-q \int \frac{d E}{2 \pi} \operatorname{Tr}\left[\bar{g}_{\beta}^{<}\left(\Sigma_{\alpha}^{a}-\bar{\Sigma}_{\alpha}^{r}\right)+\left(\bar{G}^{r} \bar{\sigma}_{\alpha}^{<}-\bar{\sigma}_{\alpha}^{<} G^{a}\right) \delta_{\alpha \beta}\right] .
$$

Here the equilibrium self-energy $\Sigma^{r} \equiv \Sigma_{L}^{r}\left(E-q V_{L}\right)+\Sigma_{R}^{r}(E$ $\left.-q V_{R}\right)$ is a $2 \times 2$ matrix in spin space, which describes the coupling of the central scattering region to the two ferromagnetic electrodes and is written as

$$
\Sigma_{\alpha}^{r}(E)=\hat{R}_{\alpha}\left(\begin{array}{cc}
\Sigma_{\alpha \uparrow}^{r} & 0 \\
0 & \Sigma_{\alpha \downarrow}^{r}
\end{array}\right) \hat{R}_{\alpha}^{\dagger}
$$

with the rotational matrix $\hat{R}_{\alpha}$ for electrode $\alpha$ defined as

$$
\hat{R}_{\alpha}=\left(\begin{array}{cc}
\cos \theta_{\alpha} / 2 & \sin \theta_{\alpha} / 2 \\
-\sin \theta_{\alpha} / 2 & \cos \theta_{\alpha} / 2
\end{array}\right) \text {. }
$$

Here angle $\theta_{\alpha}$ is defined as $\theta_{L}=0$ and $\theta_{R}=\theta$. The spin-valve effect is closely related to the magnetic field-dependent selfenergy $\Sigma_{\alpha \sigma}^{r}$ :

$$
\Sigma_{\alpha \sigma m n}^{r}=\sum_{k} T_{k \alpha m}^{*} T_{k \alpha n} /\left(E-\epsilon_{k \alpha}-\sigma M+i 0^{+}\right) .
$$


The equilibrium lesser self-energy is $\Sigma^{<}=\Sigma_{L}^{<}\left(E-q V_{L}\right)$ $+\Sigma_{R}^{<}\left(E-q V_{R}\right)$ given by the following expression at equilibrium:

$$
\Sigma_{\alpha}^{<}(E)=i f_{\alpha} \hat{R}_{\alpha}\left(\begin{array}{cc}
\Gamma_{\alpha \uparrow} & 0 \\
0 & \Gamma_{\alpha \downarrow}
\end{array}\right) \hat{R}_{\alpha}^{\dagger},
$$

where $\Gamma_{\alpha \sigma}=-2 \operatorname{Im}\left(\Sigma_{\alpha \sigma}^{r}\right)$ is the linewidth function.

To proceed further, we need the equilibrium Green's functions. We assume that in the energy range of interest there is only one single energy level with energy $E_{0}$ in the scattering region. Hence in the wideband approximation ${ }^{19}$ the matrix elements are reduced to scalars: ${ }^{14}$

$$
\begin{gathered}
G_{11}^{r}=\frac{A_{\downarrow}}{A_{\uparrow} A_{\downarrow}-B^{2}}, \\
G_{12}^{r}=G_{21}^{r}=\frac{-B}{A_{\uparrow} A_{\downarrow}-B^{2}}, \\
G_{22}^{r}=\frac{A_{\uparrow}}{A_{\uparrow} A_{\downarrow}-B^{2}},
\end{gathered}
$$

where

$$
\begin{gathered}
A_{\uparrow}=E-E_{0}+i \Gamma_{1}, \\
A_{\downarrow}=E-E_{0}+i \Gamma_{2}, \\
B=(i / 2)\left[\Gamma_{R \downarrow}-\Gamma_{R \uparrow}\right] \sin (\theta / 2) \cos (\theta / 2)
\end{gathered}
$$

and

$$
\begin{aligned}
& \Gamma_{1}=\left[\Gamma_{L \uparrow}+\cos ^{2}(\theta / 2) \Gamma_{R \uparrow}+\sin ^{2}(\theta / 2) \Gamma_{R \downarrow}\right] / 2, \\
& \Gamma_{2}=\left[\Gamma_{L \downarrow}+\cos ^{2}(\theta / 2) \Gamma_{R \downarrow}+\sin ^{2}(\theta / 2) \Gamma_{R \uparrow}\right] / 2 .
\end{aligned}
$$

The matrix elements of retarded Green functions have a simple physical meaning: the diagonal elements represent the density of states of the up-spin and down-spin separately while the nondiagonal terms are a measure of the scattering rates from up-spin to down-spin and vice versa. ${ }^{24}$ In the wideband limit, self-energy matrix elements are reduced to coupling constants that depend on the spin index

$$
\hat{\Gamma}_{L}=\left(\begin{array}{cc}
\Gamma_{L \uparrow} & 0 \\
0 & \Gamma_{L \downarrow}
\end{array}\right), \hat{\Gamma}_{R}=\left(\begin{array}{ll}
R_{1} & R_{2} \\
R_{2} & R_{3}
\end{array}\right),
$$

where

$$
\begin{aligned}
& R_{1}=\cos ^{2}(\theta / 2) \Gamma_{R \uparrow}+\sin ^{2}(\theta / 2) \Gamma_{R \downarrow}, \\
& R_{2}=\sin (\theta / 2) \cos (\theta / 2)\left[\Gamma_{R \downarrow}-\Gamma_{R \uparrow}\right], \\
& R_{3}=\cos ^{2}(\theta / 2) \Gamma_{R \downarrow}+\sin ^{2}(\theta / 2) \Gamma_{R \uparrow} .
\end{aligned}
$$

With the Green's function and self-energy already obtained, we calculate the conduction part of dynamic conductance

$$
\begin{aligned}
G_{11}^{c}(\omega)= & \frac{q^{2}}{\omega} \int \frac{d E}{2 \pi}\left[i \omega \left(\bar{G}_{11}^{r} G_{11}^{a} \Gamma_{L \uparrow}+\bar{G}_{22}^{r} G_{22}^{a} \Gamma_{L \downarrow}-\bar{G}_{12}^{r} G_{21}^{a} \Gamma_{L \uparrow}\right.\right. \\
& \left.-\bar{G}_{21}^{r} G_{12}^{a} \Gamma_{L \downarrow}\right)+\left(-\bar{G}_{11}^{r} G_{11}^{a} \Gamma_{L \uparrow} R_{1}-\bar{G}_{22}^{r} G_{22}^{a} \Gamma_{L \downarrow} R_{3}\right. \\
& \left.\left.+\bar{G}_{12}^{r} G_{21}^{a}\left(2 \Gamma_{L \downarrow}+R_{3}\right) \Gamma_{L \uparrow}+\bar{G}_{21}^{r} G_{12}^{a}\left(2 \Gamma_{L \uparrow}+R_{1}\right) \Gamma_{L \downarrow}\right)\right] \\
& \times(f-\bar{f})
\end{aligned}
$$

and

$$
\begin{aligned}
G_{12}^{c}(\omega)= & \frac{q^{2}}{w} \int \frac{d E}{2 \pi}\left[\bar{G}_{11}^{r} G_{11}^{a} \Gamma_{L \uparrow} R_{1}+\bar{G}_{22}^{r} G_{22}^{a} \Gamma_{L \downarrow} R_{3}+\bar{G}_{12}^{r} G_{21}^{a}\right. \\
& \times\left(-2 \Gamma_{L \downarrow}-R_{3}+2 i w\right) \Gamma_{L \uparrow}+\bar{G}_{21}^{r} G_{12}^{a}\left(-2 \Gamma_{L \uparrow}-R_{1}\right. \\
& \left.+2 i w) \Gamma_{L \downarrow}\right](f-\bar{f})
\end{aligned}
$$

and the contribution from displacement current

$$
\begin{aligned}
G_{1}^{d}(\omega)= & -i q^{2} \int \frac{d E}{2 \pi}\left(G_{11}^{r} G_{11}^{a} \Gamma_{L \uparrow}+\bar{G}_{22}^{r} G_{22}^{a} \Gamma_{L \downarrow}+\bar{G}_{12}^{r} G_{21}^{a} \Gamma_{L \downarrow}\right. \\
& \left.+\bar{G}_{21}^{r} G_{12}^{a} \Gamma_{L \uparrow}\right)(f-\bar{f})
\end{aligned}
$$

and

$$
\begin{aligned}
G_{2}^{d}(\omega)= & -i q^{2} \int \frac{d E}{2 \pi}\left[\bar{G}_{11}^{r} G_{11}^{a} R_{1}+\bar{G}_{22}^{r} G_{22}^{a} R_{3}+\bar{G}_{12}^{r} G_{21}^{a}\left(-2 \Gamma_{L \downarrow}\right.\right. \\
& \left.\left.-R_{3}+2 i \omega\right)+\bar{G}_{21}^{r} G_{12}^{a}\left(-2 \Gamma_{L \uparrow}-R_{1}+2 i \omega\right)\right](f-\bar{f}) .
\end{aligned}
$$

In all subsequent analysis we will deal with zero-temperature phenomena and so the energy integration will be from $E$ $=E_{f}$ to $E_{f}-\omega$.

\section{NUMERICAL RESULTS}

Now we present the numerical calculations of the dynamic conductance as different external parameters vary. Since the Buttiker's formalism is current conserving and gauge-invariant, the dynamic conductance $G_{\alpha \beta}$ satisfies $\Sigma_{\alpha} G_{\alpha \beta}=0$ and $\Sigma_{\beta} G_{\alpha \beta}=0$. As a result, there is only one independent coefficient. In the following, we choose to calculate $G=G_{11}=-G_{12}$ because its real part is positive and corresponds to a (positive) measurable quantity. In the dc limit, $G$ is a real quantity. As frequency is turned on, it becomes a complex quantity with real part $G_{R}$ which is related to the dissipation and imaginary part $G_{I}$ describing the phase difference between current and voltage. In the subsequent calculation, we will fix $E_{f}=E_{0}=5 \mathrm{meV}$ and investigate the dynamic conductance for the Fermi energy at resonance. For simplicity, we only consider the symmetric case that $\Gamma_{L \sigma}$ $=\Gamma_{R \sigma}=\Gamma_{\sigma}$ and that $\Gamma_{\uparrow} \geqslant \Gamma_{\downarrow}$ and set $\Gamma_{\uparrow}=0.5 \mathrm{meV}$ and $\Gamma_{\downarrow}$ $=0.1 \mathrm{meV}$ unless otherwise specified.

The angular dependence of $G_{R}(\theta, \omega)$ at different frequencies $\omega$ is shown in Fig. 1. We see that at small frequencies $\omega=0.05 \mathrm{meV}, G_{R}$ has a minimum at $\theta=\pi$ and is peaked at $\theta=0($ or $2 \pi)$. As the frequency increases, the minimum of $G_{R}$ does not change much whereas its maximum value decreases quickly. This can be understood as follows. In the dc case, only the electron on the Fermi level contributes to the con- 


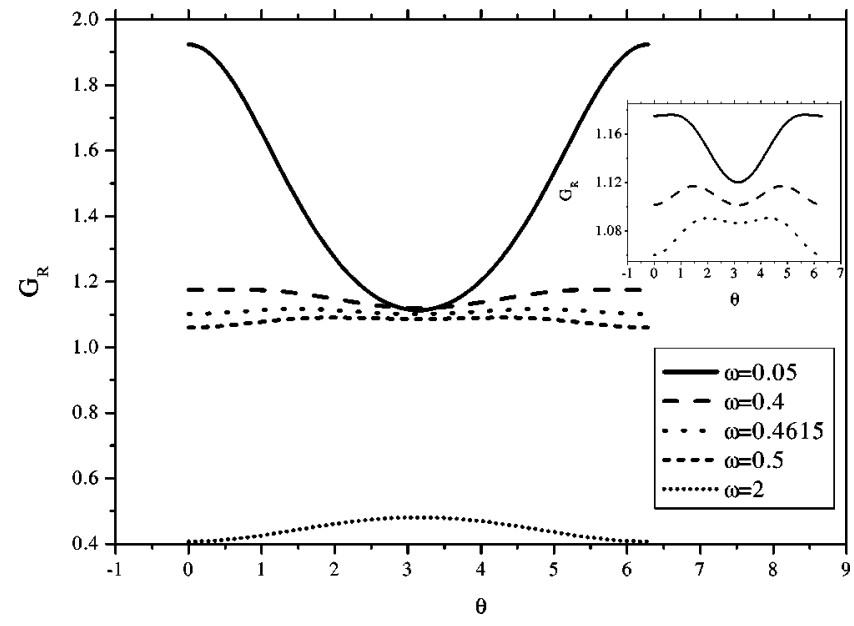

FIG. 1. The real part of dynamic conductance $G_{R}$ vs angle $\theta$ at different frequencies. The solid, dashed, dotted, short-dashed, and short-dotted lines correspond to $\omega=0.05,0.4,0.4615,0.5$, and $2.0 \mathrm{meV}$, respectively. Inset: amplified feature of $G_{R}$ vs $\theta$ at $\omega$ $=0.4,0.4615,0.5 \mathrm{meV}$ (solid line, dashed line, and dotted line).

ductance. In the ac case, the electrons with energy $\left[E_{F}\right.$ $\left.-\hbar \omega, E_{F}\right]$ can absorb a photon and participate the transport process. This photon assisted tunneling is reflected in the weighting factor $(f-\bar{f}) / \omega$ in Eq. (21) which gives $-d f / d E$ in the dc limit. Because of this inelastic channel, the resonant feature is smeared off. As a result, the dynamic conductance decreases near the resonance as the frequency is increased. When the frequency is around $\omega=0.4 \mathrm{meV}$, two maxima for $G_{R}$ occur at $\theta=0.8$ and 5.2 (see the inset of Fig. 1). As the frequency increases further, two maxima move towards each other and their magnitude decrease. When $\omega=0.5 \mathrm{meV}$, two maximum start to merge and the minimum at $\theta=\pi$ gradually turns to a maximum. From this figure, we conclude that the maximum of $G_{R}$ can occur at different angles if the frequency is modulated. As frequency is varied, $G_{R}$ changed its convexity from concave at $\omega=0.05 \mathrm{meV}$ to convex at $\omega>0.5 \mathrm{meV}$. The imaginary part of the dynamic conductance $G_{I}$ as a function of $\theta$ is depicted in Fig. 2 at different frequencies. We see that the shape of $G_{I}$ is concave for all frequencies. In addition, $G_{I}$ 's are positive indicating inductivelike response of the system. ${ }^{25,26}$

To discuss the spin-valve effect we examine the tunneling magnetoresistance (TMR) ratio defined as $\left[G_{R}(0)\right.$ $\left.-G_{R}(\pi)\right] / G_{R}(0)$ (Ref. 12) which can be calculated analytically as follows. From Eqs. (7) and (9) we obtain

$$
\begin{gathered}
G(0)=-\frac{i \Gamma_{\uparrow}-2 \Gamma_{\uparrow}^{2} / \omega}{2 i \Gamma_{\uparrow}+\omega}\left[\ln \Gamma_{\uparrow}-\ln \left(i w-\Gamma_{\uparrow}\right)\right] \\
-\frac{i \Gamma_{\downarrow}-2 \Gamma_{\downarrow}^{2} / \omega}{2 i \Gamma_{\downarrow}+\omega}\left[\ln \Gamma_{\downarrow}-\ln \left[\left(i w-\Gamma_{\downarrow}\right)\right]\right], \\
G(\pi)=-\frac{i \Gamma-4 \Gamma_{\uparrow} \Gamma_{\downarrow} / \omega}{i \Gamma+\omega}[\ln (\Gamma / 2)-\ln [(i w-\Gamma / 2)]],
\end{gathered}
$$

where $\quad \Gamma=\Gamma_{\uparrow}+\Gamma_{\downarrow}$. Note that $\ln (a+i b)=\left[\ln \left(a^{2}+b^{2}\right)\right] / 2$ $+i \tan ^{-1}(b / a)$. The real part of the above equations yield

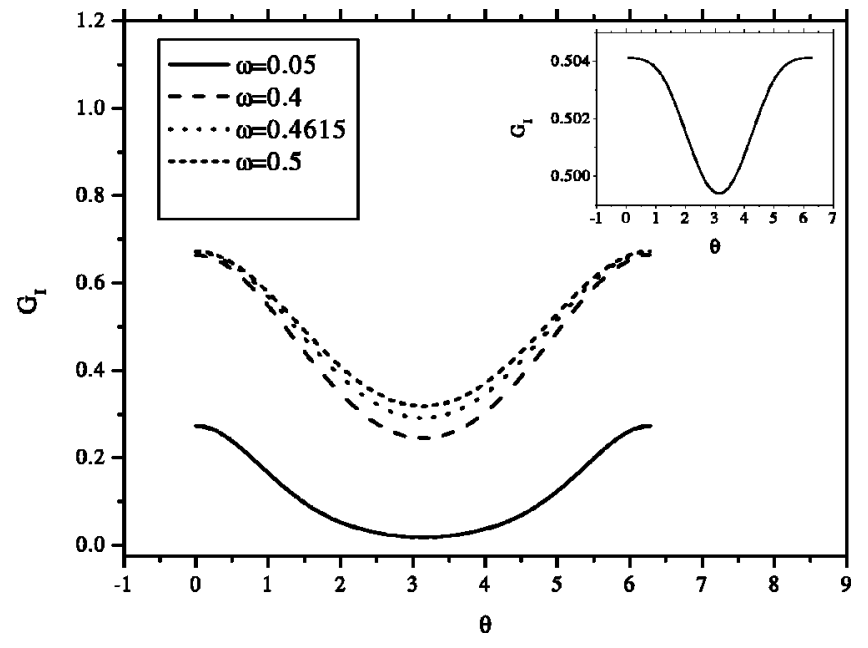

FIG. 2. The imaginary part of dynamic conductance $G_{I}$ vs $\theta$ at different frequencies. The solid, dashed, dotted, short-dashed lines correspond to $\omega=0.05,0.40,0.4615$, and $0.5 \mathrm{meV}$, respectively. Inset: $G_{I}$ vs $\theta$ at $\omega=2.0 \mathrm{meV}$.

$$
\begin{aligned}
G_{R}(0)= & -\left(\Gamma_{\uparrow} / 2 \omega\right)\left[2 \tan ^{-1}\left(\Gamma_{\uparrow} / \omega\right)-\pi\right]-\left(\Gamma_{\downarrow} / 2 \omega\right) \\
& \times\left[2 \tan ^{-1}\left(\Gamma_{\downarrow} / \omega\right)-\pi\right]
\end{aligned}
$$

and

$$
\begin{aligned}
G_{R}(\pi)= & -\frac{\left(\Gamma_{\uparrow}-\Gamma_{\downarrow}\right)^{2}\left[\ln \left(\Gamma^{2} / 4\right)-\ln \left(\omega^{2}+\Gamma^{2} / 4\right)\right]}{2\left(\omega^{2}+\Gamma^{2}\right)} \\
& -\frac{\Gamma\left(\omega / 2+2 \Gamma_{\uparrow} \Gamma_{\downarrow} / \omega\right)\left[2 \tan ^{-1}(\Gamma / 2 \omega)-\pi\right]}{\omega^{2}+\Gamma^{2}} .
\end{aligned}
$$

In the low-frequency limit, we have

$$
\begin{gathered}
G_{R}(0)=2+O\left(\omega^{2}\right), \\
G_{R}(\pi)=8 \Gamma_{\uparrow} \Gamma_{\downarrow} / \Gamma^{2}+O\left(\omega^{2}\right)
\end{gathered}
$$

which agrees with the dc limit in Ref. 14. From Eq. (24), we conclude that $G_{R}(0)$ is always greater than $G_{R}(\pi)$ regardless of the choices of $\Gamma_{\uparrow}$ and $\Gamma_{\downarrow}$. In the high-frequency limit, we expand Eq. (23) in terms of $1 / \omega$ up to the second order

$$
\begin{gathered}
G_{R}(0)=\frac{\Gamma \pi}{2 \omega}-\frac{\left(\Gamma_{\uparrow}^{2}+\Gamma_{\downarrow}^{2}\right)}{2 \omega^{2}}, \\
G_{R}(\pi)=\frac{\Gamma \pi}{2 \omega}+\frac{\left(\Gamma_{\uparrow}-\Gamma_{\downarrow}\right)^{2} \ln \omega}{\omega^{2}}-\frac{\Gamma^{2}}{2 \omega^{2}} .
\end{gathered}
$$

Hence in the large frequency limit, $G_{R}(\pi)$ is larger than $G_{R}(0)$. This explains is the change in convexity of $G_{R}$ shown in Fig. 1. From Eqs. (22) and (23), the TMR ratio is shown in Fig. 3. We see that as the frequency is turned on the TMR ratio drops sharply, goes to zero at $\omega_{c}=0.46 \mathrm{meV}$, and becomes negative when $\omega>\omega_{c}$. The change of the TMR ratio from positive to negative cannot happen in the dc case because the conductance when magnetizations of two leads are parallel is always larger than that of antiparallel case, i.e., the TMR ratio is positive definite. We note that the negative 


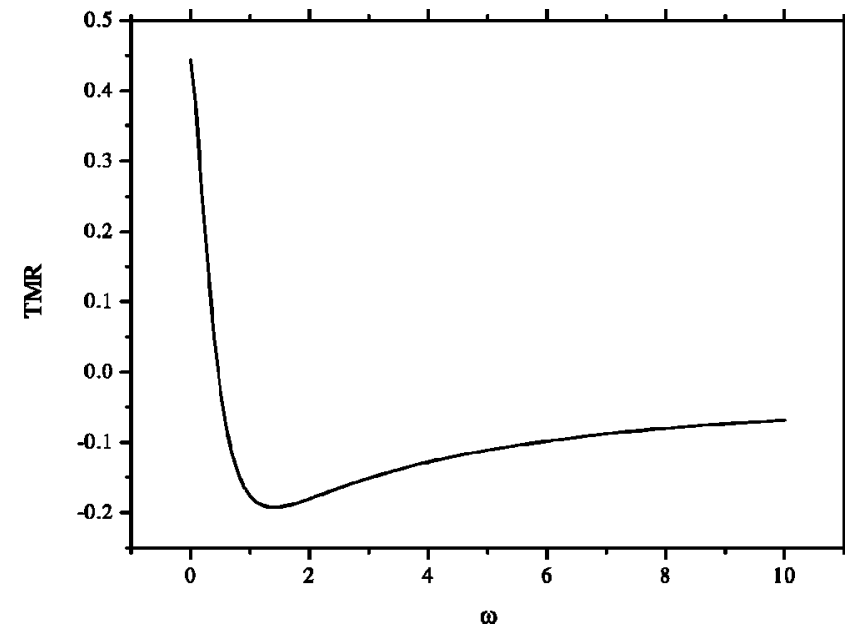

FIG. 3. The TMR ratio vs frequency $\omega$.

TMR ratio can also occur in FM/I/FM/I/FM double barrier magnetic layered structures which is controlled by the bias voltage. ${ }^{12}$ The physics behind the transition from positive TMR to negative TMR due to an increase in frequency is the following. In the dc case, when $\theta=0$ the tunnelings for electrons with both spins are through symmetric system whereas for $\theta=\pi$ the tunneling processes are asymmetric. Hence near the resonance $E \sim E_{0}$, we have $G_{R}(0, \omega=0)>G_{R}(\pi, \omega=0)$ or a positive TMR. In a way, $E \sim E_{0}$ for $\theta=\pi$ is almost off resonance for $\theta=0$. As pointed out by Annatram and Datta ${ }^{22}$ that on the resonance the dynamic conductance decreases much faster that off the resonance as the frequency is turned on leading to a negative TMR. The generalized TMR ratio $\left[G_{R}(0)-G_{R}(\theta)\right] / G_{R}(0)$ at different frequency vs $\theta$ is shown in Fig. 4 which resembles the behaviors in Fig. 1. It is also interesting to vary the ratio of the spin-dependent coupling constants $\kappa=\Gamma_{\uparrow} / \Gamma_{\downarrow}$ and examine how the corresponding TMR ratio [denoted as $\operatorname{TMR}(\kappa)$ ] changes. In Fig. 5, TMR $(\kappa)$ is plotted at a fixed $\Gamma_{\downarrow}=0.1$. Since $G(0)=G(\pi)$ when $\Gamma_{\uparrow}$

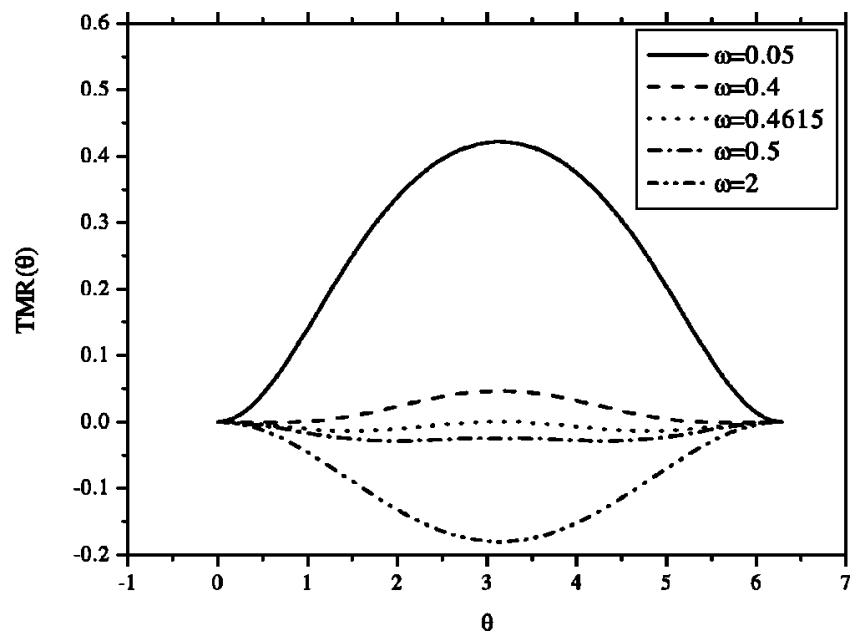

FIG. 4. The generalized TMR ratio $\operatorname{TMR}(\theta)$ vs $\theta$ at different frequencies. The solid, dashed, dotted, short-dashed, and shortdotted lines correspond to $\omega=0.05,0.4,0.4615,0.5$, and $2.0 \mathrm{meV}$, respectively.

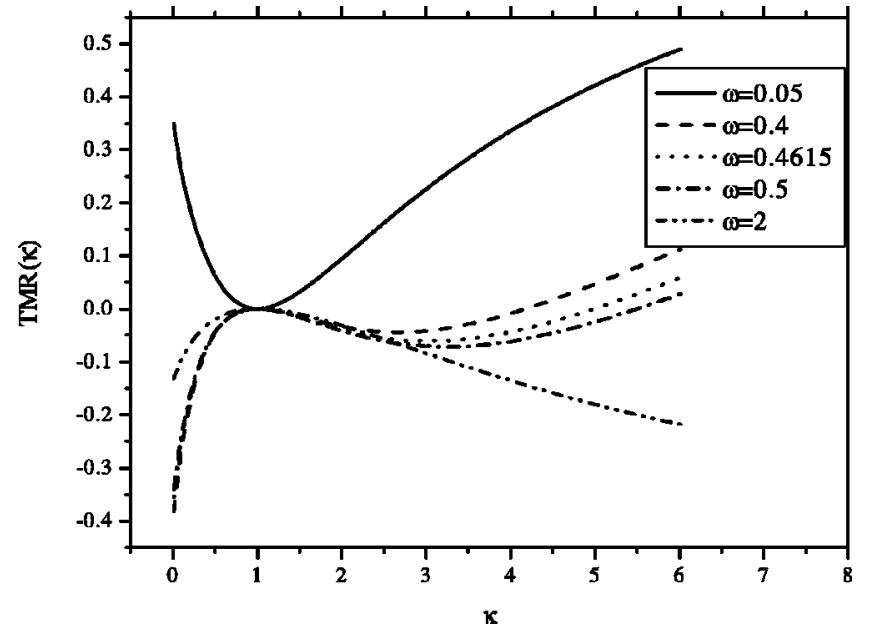

FIG. 5. The TMR ratio vs $\kappa$ at different frequencies. The solid, dashed, dotted, short-dashed, and short-dotted lines correspond to $\omega=0.05,0.4,0.4615,0.5$, and $2.0 \mathrm{meV}$, respectively.

$=\Gamma_{\downarrow}$, the TMR ratio vanishes at $\kappa=1$. In general, the further away $\kappa$ from one, the larger TMR ratio is.

Let us now focus on $G_{R}$ as a function of the Fermi energy at two different frequencies $\omega=0.05,2 \mathrm{meV}$. We see from Fig. 6(a) that at small frequency $\omega=0.05 \mathrm{meV}, G_{R}$ shows the resonant behavior for both $\theta=0$ and $\pi$ with Lorentz-like line shape. The width of the resonant peaks are determined by the larger linewidth function $\Gamma_{\uparrow}$. At much larger frequency $\omega$ $=2 \mathrm{meV}$ [inset of Fig. 6(a)], the line shapes deviate significantly away from Lorentzian. In addition, the peaks of $G_{R}$ are much broader with much smaller peak values. In Fig. 6(b), we plot the imaginary part of dynamic conductance $G_{I}$. At small frequency $\omega=0.05 \mathrm{meV}, G_{I}$ at $\theta=0$ shows typical resonant feature. ${ }^{25,26}$ At the resonance $E=E_{0}$, the system conducts very well and shows inductivelike behavior with positive $G_{I}$ and when the energy is away from the resonance the capacitivelike behavior dominates and $G_{I}$ turns to negative. At $\theta=\pi$, the system is less transmissive at $E=E_{0}$ so that the peak of $G_{I}$ is much smaller. At larger frequency [inset of Fig. 6(b)], similar behaviors are observed.

The behavior of $G_{R}$ and $G_{I}$ at small $\omega$ can also be understood qualitatively from a classical circuit model. ${ }^{27}$ Due to both inductivelike and capacitivelike responses, our system can be considered as an inductor in series with a parallel connection of a capacitor and a resistor. For this classical circuit the dynamic conductance can be written ${ }^{27}$ in the following form in the small frequency limit $\left(e^{2} / h=1\right)$ :

$$
G(\omega)=\frac{1}{R}-i \omega\left[C-\frac{L}{R^{2}}\right]+\omega^{2} \frac{L}{R}\left(2 C-\frac{L}{R^{2}}\right) .
$$

The term linear in $\omega$, which corresponds to $G_{I}$, shows a competition between two different dynamic responses. If $C>L / R^{2}$, the response is capacitivelike with a negative $G_{I}$. A similar argument for $G_{I}$ applies to the inductivelike region when $C<L / R^{2}$. On the other hand, the dissipative component $G_{R}$, near the resonance, can increase or decrease with $\omega$ depending on the sign of the term of order $\omega^{2}$ in Eq. (26). According to this picture $G_{R}$ decreases when $C<L /\left(2 R^{2}\right)$ 

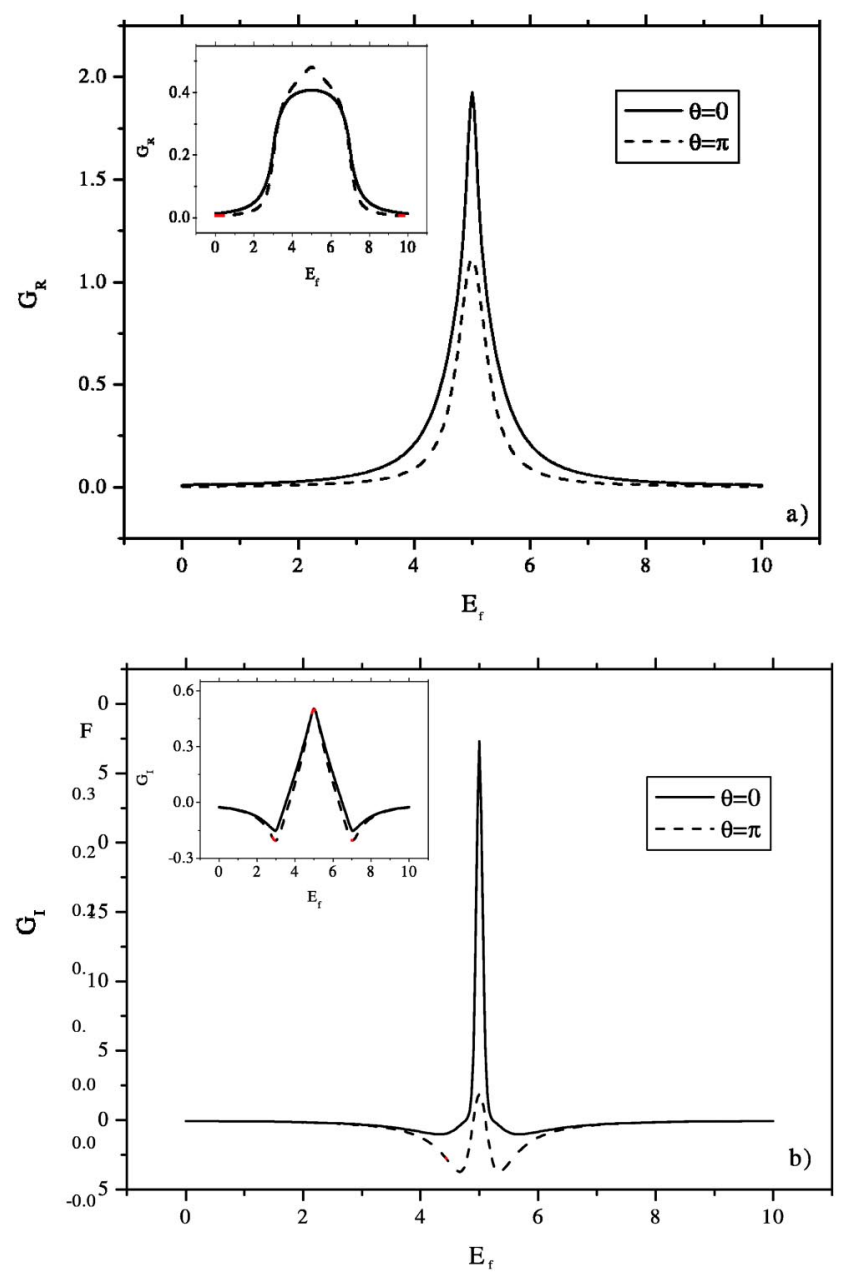

FIG. 6. The dynamic conductance vs the Fermi energy when $\theta=0, \pi$ (a). $G_{R}$ vs $E$. Main panel: $\omega=0.05 \mathrm{meV}$. Inset: $\omega$ $=2.0 \mathrm{meV}$. (b). $G_{I}$ vs $E$. Main panel: $\omega=0.05 \mathrm{meV}$. Inset: $\omega$ $=2.0 \mathrm{meV}$.

and increases otherwise. In particular, for the transport through a single resonant level, the system is very transmissive so that the capacitance $C \sim 0$. Hence near the resonance, the real part of the dynamic conductance $G_{R}$ decreases when the frequency is switched on as we have observed in Fig. 1. Note, however, that when the frequency is so large that $\omega^{3}$ or higher order terms cannot be neglected, Eq. (26) breaks down. To relate the classical circuit model to our system we expand the ac conductance coefficients $G_{R}(0)$ and $G_{R}(\pi)$ up to the second order in frequency. By comparing the coefficients of expansion with Eq. (26) and noting that $1 / R$ $=G_{R}(\omega=0)$ we obtain the following results at $\theta=0$ :

$$
\begin{gathered}
R=\frac{1}{2}, \\
C=\sqrt{\frac{5}{4}\left(\frac{1}{\Gamma_{\uparrow}^{2}}+\frac{1}{\Gamma_{\downarrow}^{2}}\right)+\frac{1}{2} \frac{1}{\Gamma_{\uparrow} \Gamma_{\downarrow}}}, \\
L=\frac{C}{2}+\frac{1}{2}\left(\frac{1}{\Gamma_{\uparrow}}+\frac{1}{\Gamma_{\downarrow}}\right) .
\end{gathered}
$$
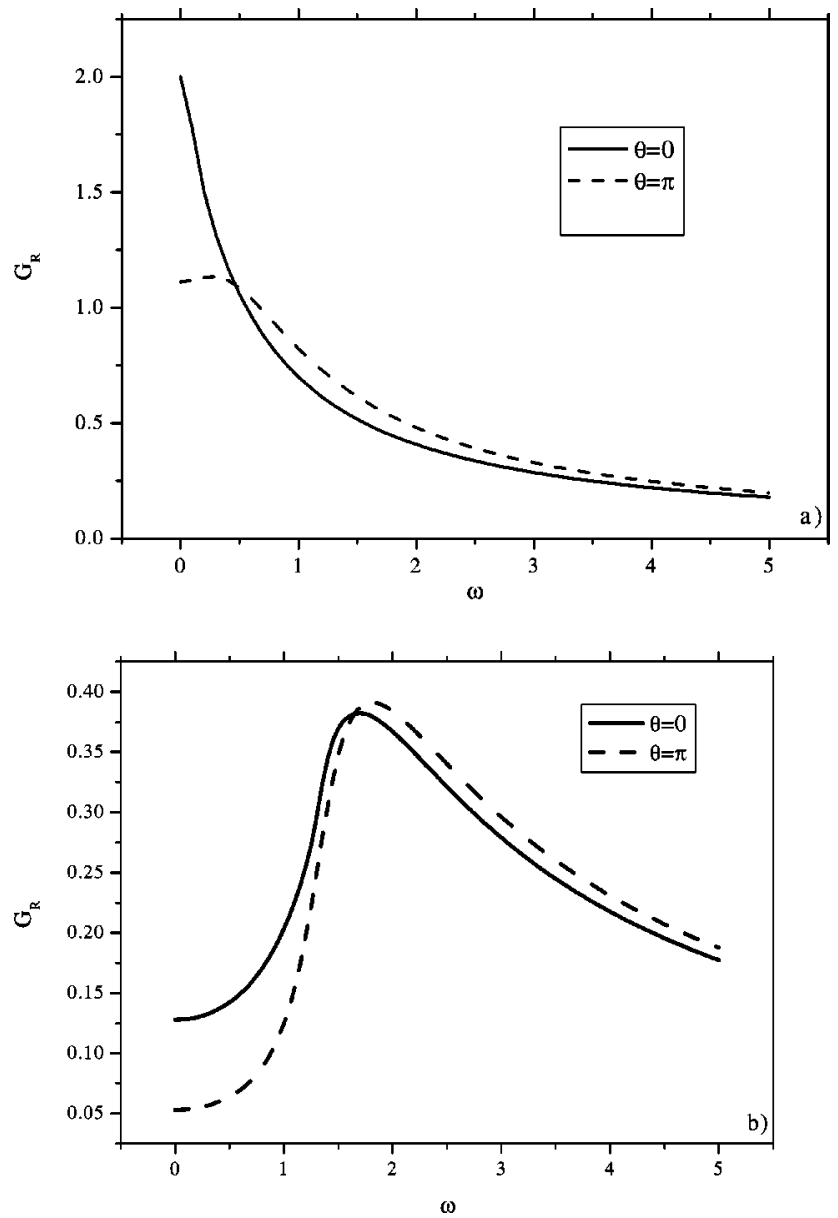

FIG. 7. $G_{R}$ vs $\omega$ at $\theta=0$ (solid line) and $\pi$ (dashed line). (a) $E=E_{0}$, (b) $E=E_{0}+1.34 \mathrm{meV}$.

For $\Gamma_{\uparrow}=0.5 \mathrm{meV}$ and $\Gamma_{\downarrow}=0.1 \mathrm{meV}$, we have $C=11.8 / \mathrm{meV}$ and $L=11.9 / \mathrm{meV}$ (note that the unit of the conductance is $e^{2} / h$ has been set to 1$)$. While for $\theta=\pi$ the corresponding parameters are

$$
\begin{gathered}
R=\frac{\Gamma^{2}}{2 \Gamma_{\uparrow} \Gamma_{\downarrow}}, \\
C=\sqrt{\left(\frac{5}{\Gamma}-\frac{\Gamma}{\Gamma_{\uparrow} \Gamma_{\downarrow}}\right)^{2}+\frac{2}{\Gamma_{\uparrow} \Gamma_{\downarrow}}}, \\
L=\frac{C \Gamma^{2}}{2 \Gamma_{\uparrow} \Gamma_{\downarrow}}+\frac{5}{\Gamma}-\frac{\Gamma}{\Gamma_{\uparrow} \Gamma_{\downarrow}} .
\end{gathered}
$$

For $\Gamma_{\uparrow}=0.5 \mathrm{meV}$ and $\Gamma_{\downarrow}=0.1 \mathrm{meV}$, we have $R=3.6, C$ $=7.3 / \mathrm{meV}$ and $L=22.6 / \mathrm{meV}$.

To get more information, $G_{R}$ versus frequency for $\theta=0, \pi$ is plotted in Fig. 7 when $E=E_{0}$. Denote $G_{R}(0)=G_{R}(\theta=0)$. We see that $G_{R}(0)$ drops quickly when $\omega<1$ and saturate at larger frequencies whereas $G_{R}(\pi)$ is flat for frequency between $[0,0.5] \mathrm{meV}$ and then decreases in a similar fashion as $G_{R}(0)$. Note that $G_{R}(0)$ is greater than $G_{R}(\pi)$ only when $\omega<0.5 \mathrm{meV}$. In Fig. 7(b), we plot $G_{R}$ vs $\omega$ when the system is off resonance $E=E_{0}+1.34 \mathrm{meV}$. We see that $G_{R}$ increases 

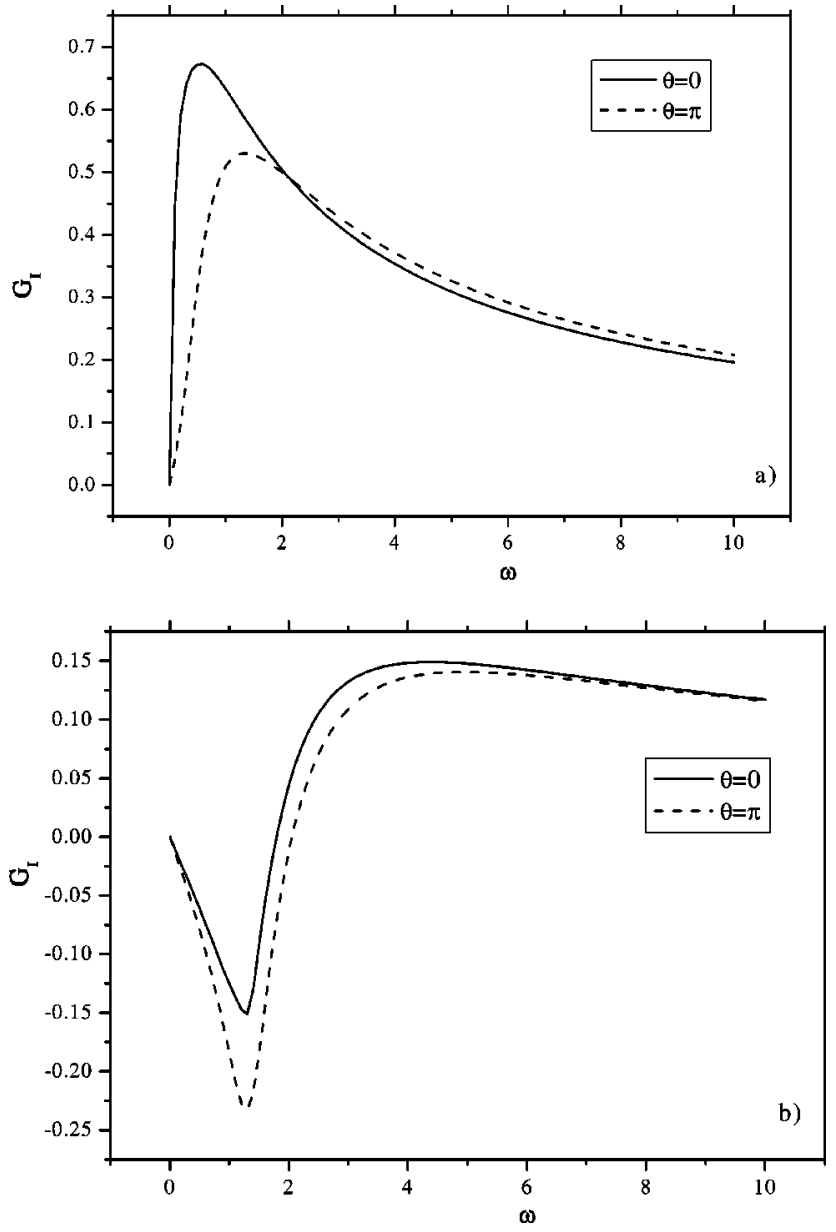

FIG. 8. $G_{I}$ vs $\omega$ when $\theta=0, \pi$. (a) $E=E_{0}$, (b) $E=E_{0}$ $+1.34 \mathrm{meV}$. upon increasing the frequency. This is consistent with Eq. (26) because when off the resonance the system is capacitivelike. Figure 8 shows and $G_{I}$ vs frequency at different energies. We see that on the resonance $E=E_{0}, G_{I}$ is always positive. As the frequency is turned on, $G_{I}$ increases quickly and reaches maximum. Upon further increasing the frequency, $G_{I}$ decreases slowly. When off the resonance, however, $G_{I}$ becomes negative at small frequencies and then turns to positive values at large frequencies.

In conclusion, we have studied spin valve effect through a resonant level between two ferromagnetic leads in the presence of an ac voltage. In particular, we have investigated the dependence of the dynamic conductance on various parameters such as the angle between magnetizations of two leads $\theta$, the ac frequency $\omega$, and spin-dependent coupling constants $\Gamma_{\sigma}$. We found that the TMR ratio can be modulated by ac frequency and the negative magnetoresistance can occur at large frequency. The frequency range considered in this work is in the $\mathrm{THz}$ regime which is within the reach of present technology. ${ }^{28}$ Our theory is suitable for describing noninteracting systems which is phase coherent and operating at a small bias. Finally, our theory can be extended to the cases such as the Coulomb-blockade regime in double barrier $\mathrm{F}-\mathrm{N}-\mathrm{F}$ systems $^{29}$ and the inclusion of elementary excitations similar to phonons and magnons. ${ }^{30}$

\section{ACKNOWLEDGMENTS}

We gratefully acknowledge financial support from RGC Grant No. HKU 7113/02P from the government of Hong Kong SAR and a grant from CRCG of The University of Hong Kong. B.G.W. thanks the NSFC for financial support under Grant No. 90303011.
*Electronic address: jianwang@hkusub.hku.hk

${ }^{1}$ M. Julliere, Phys. Lett. 54A, 225 (1975).

${ }^{2}$ J. C. Slonczewski, Phys. Rev. B 39, 6995 (1989).

${ }^{3}$ J.S. Moodera, L.R. Kinder, T.M. Wong, and R. Meservey, Phys. Rev. Lett. 74, 3273 (1995); J.S. Moodera, J. Nowak, and R.J. M. van de Veerdonk, ibid. 80, 2941 (1998).

${ }^{4}$ X.D. Zhang, B.Z. Li, G. Sun, and F.C. Pu, Phys. Rev. B 56, 5484 (1997)

${ }^{5}$ A.M. Bratkovsky, Phys. Rev. B 56, 2344 (1997).

${ }^{6}$ J. Barnaś and A. Fert, Phys. Rev. Lett. 80, 1058 (1998).

${ }^{7}$ S. Zhang, P.M. Levy, A.C. Marley, and S.S.P. Parkin, Phys. Rev. Lett. 79, 3744 (1997).

${ }^{8}$ J. Inoue and S. Maekawa, Phys. Rev. B 53, R11 927 (1996).

${ }^{9}$ J.M. Maclaren, X.-G. Zhang, and W.H. Butler, Phys. Rev. B 56, 11827 (1997).

${ }^{10}$ J. Mathon, Phys. Rev. B 56, 11810 (1997).

${ }^{11}$ A. Brataas, Yu. V. Nazarow, J. Inoue, and G.E.W. Bauer, Phys. Rev. B 59, 93 (1999).

${ }^{12}$ L. Sheng, Y. Chen, H.Y. Teng, and C.S. Ting, Phys. Rev. B 59, 480 (1999).

${ }^{13}$ H. Mehrez, J. Taylor, H. Guo, J. Wang, and C. Roland, Phys. Rev.
Lett. 84, 2682 (2000).

${ }^{14}$ B.G. Wang, J. Wang, and H. Guo, J. Phys. Soc. Jpn. 70, 2654 (2001).

${ }^{15}$ N. Sergueev, Q.F. Sun, H. Guo, and J. Wang, Phys. Rev. B 65, 165303 (2002).

${ }^{16}$ F. Mireles and G. Kirczenow, Phys. Rev. B 66, 214415 (2002).

${ }^{17}$ Z.C. Wang, G. Su, and Q.R. Zheng, Phys. Rev. B 64, 014407 (2001).

${ }^{18}$ M. Büttiker, A. Prêtre, and H. Thomas, Phys. Rev. Lett. 70, 4114 (1993).

${ }^{19}$ A.P. Jauho, N.S. Wingreen, and Y. Meir, Phys. Rev. B 50, 5528 (1994).

${ }^{20}$ D.C. Langreth, in Linear and Nonlinear Electron Transport in Solids, edited by J.T. Devreese and E. Van Doren (Plenum, New York, 1976).

${ }^{21}$ The calculation is very similar to that of Ref. 22 .

${ }^{22}$ P.A. Anantram and S. Datta, Phys. Rev. B 51, 7632 (1995).

${ }^{23}$ B.G. Wang, J. Wang, and H. Guo, Phys. Rev. Lett. 82, 398 (1999).

${ }^{24}$ S. Datta, Electronic Transport in Mesoscopic Systems (Cambridge University Press, New York, 1995). 
${ }^{25}$ M. Büttiker, H. Thomas, and A. Prétre, Z. Phys. B: Condens. Matter 94, 133 (1994).

${ }^{26}$ W. Zheng, Y.D. Wei, J. Wang, and H. Guo, Phys. Rev. B 61, 13121 (2000).

${ }^{27}$ A. Prêtre, H. Thomas, and M. Büttiker, Phys. Rev. B 54, 8130
(1996).

${ }^{28}$ P.S. S. Guimaraes et al., Phys. Rev. Lett. 70, 3792 (1993).

${ }^{29}$ G. Usaj and H.U. Baranger, Phys. Rev. B 63, 184418 (2001).

${ }^{30}$ A.M. Bratkovsky, Appl. Phys. Lett. 72, 2334 (1998). 